\title{
Editorial
}

\section{Advanced Infrared Technology and Applications}

\author{
Ovidio Salvetti, ${ }^{1}$ Laura Abbozzo Ronchi, ${ }^{2}$ Carlo Corsi, ${ }^{3}$ \\ Antoni Rogalski, ${ }^{4}$ and Marija Strojnik ${ }^{5}$ \\ ${ }^{1}$ Institute of Information Science and Technologies, National Research Council of Italy, Pisa, Italy \\ ${ }^{2}$ Giorgio Ronchi Foundation, Florence, Italy \\ ${ }^{3}$ Electro-Optics Research Center, C.R.E.O., L'Aquila, Italy \\ ${ }^{4}$ Institute of Applied Physics, Military University of Technology, Warsaw, Poland \\ ${ }^{5}$ Center for Research in Optics, Leon, GTO, Mexico
}

Correspondence should be addressed to Ovidio Salvetti; ovidio.salvetti@isti.cnr.it

Received 13 January 2013; Accepted 13 January 2013

Copyright ( 2013 Ovidio Salvetti et al. This is an open access article distributed under the Creative Commons Attribution License, which permits unrestricted use, distribution, and reproduction in any medium, provided the original work is properly cited.

Nowadays, advanced infrared techniques are a key ingredient in disparate applications, ranging from medical diagnostics and treatment to industrial inspection and environmental monitoring. We believe that the success of such infrared applications highly depends on a continuous exchange between scientific advances and technological progresses.

After the success of the 11th International Workshop Advanced Infrared Technology and Applications (AITA) held in L'Aquila (Italy), September 2011, it was then decided to organize this special issue in order to further foster such synergy by assessing the state of the art of the technology in the near, mid, and far Infrared and by presenting its most interesting and innovative applications.

AITA workshop, an event of a biennial series started in 1991 and organized by the "Giorgio Ronchi" Foundation (Florence), the Institutes of Information Science and Technologies "Alessandro Faedo" (Pisa), Construction Technologies (Padova), Applied Physics "Nello Carrara" (Florence) of the National Research Council of Italy and C.R.E.O. Consortium L'Aquila, constitutes an international forum bringing together academic and industrial researchers to exchange knowledge, ideas, and experiences in the field of infrared science and technology.

Main topics of the workshop included in particular advanced technology and materials, smart and fiber-optic sensors, aerospace and industrial applications, astronomy and earth monitoring, nondestructive tests and evaluation, systems for cultural heritage, near-, mid-, and long wavelength systems, and image processing and data analysis.
The special issue was not only intended to collect extended version of selected papers presented at the workshop, but submission was open to every investigator in the field, with the aim to have an even wider coverage of all advances in this area.

This special issue includes fourteen papers which discuss scientific and technological aspects related to these areas.

In the area of advanced sensors, C. Corsi illustrates the highlights of the main historical IR developments and their impact in security applications and, correlated with the leading technology of Silicon Microelectronics, scientific and economic comparisons are discussed.

M. Kondow and F. Ishikawa study reproducible growth of high-quality GaInNAs by molecular beam epitaxy, while a chemical warfare agents analyzer is introduced by C. Corsi et al. where the authors propose low cost, room temperature, micro-bolometer sensors.

Finally, J. Wrobel et al. analyze some of the consequences of omitting series resistance in InAs/GaSb p-i-n T2SL photodiode dark current modeling, using simplified $p-n$ junction model. As is shown, incorrect series resistance value might cause discrepancies between generation/recombination and diffusion effective carrier lifetimes over one order of magnitude.

In the area of advanced technology and materials, M. D'Acunto et al. propose a single molecule detection based on near-infrared sensors and discuss biomedical applications. In particular, they study how the optical properties of gold nanoparticles and core-gold nanoshells are changed 
as a function of different sizes, shapes, composition, and biomolecular coating with characteristic shifts towards nearinfrared region. J. Tesar et al. instead consider improvement procedures of surface properties such as laser hardening which are becoming more and more important in present manufacturing and discuss methods for their analysis through infrared imaging.

In the area of image processing and data analysis, Strojnik and G. Paez propose and demonstrate the performance of an optical/IR double-image setup capable to observe rapidly varying phenomena up to 1000 frames per second. Applications to flame analysis for combustion efficiency are given.

In Morikawa et al., the applicability of the uncooled microbolometer to the microscale thermal analysis on phase transitions of organic and polymeric materials is discussed in comparison with the photon detector. Their study is motivated by the fact that noncontact thermal imaging methods are preferred in characterization of materials in increasing number of applications. In particular, creating and monitoring thermal distributions with a spatial resolution of $\sim 10 \mu \mathrm{m}$ is required for the materials developed in the energy saving and renewable technology.

The paper by D. Balageas starts with the observation that active thermography can give the possibility to characterize thermophysical properties and defects in complex structures, but that pixel-wise data processing based on 1-D model is the only reasonable approach for rapid image processing, due to the size of modern thermographic images. It is then argued that the only way to conciliate these two constraints when dealing with time-resolved experiments lies in the earlier possible detection/characterization. This approach is illustrated by several different applications and compared to more classical methods, demonstrating that simplicity of models and calculations is compatible with efficient and accurate identifications.

The area of Infrared-based nondestructive evaluation and industrial applications collects three contributions. C. Meola et al. present a method for monitoring composites under cyclic bending tests by means of infrared thermography. They focus on three types of composites including an epoxy matrix reinforced with either carbon fibers or glass fibers and hybrid composite involving glass fibers and aluminum layers. C. Toscano et al. introduce thermography methods for detecting porosity and inclusions in carbon fiber reinforced polymers, widely used in the aeronautical industry. More precisely, they consider both lock-in and pulse thermography in the transmission mode and conclude that both the techniques are definitely able to reveal the presence of the defects above mentioned; nevertheless, the techniques could be considered complementary in order to better assess the nature of defects.

Emissivity measurement of semitransparent textile is addressed by $\mathrm{P}$. Bison et al., where the authors compare three textiles, namely one normal and two "special" with $\mathrm{Ag}^{+}$ ions and carbon powder added, with different colors. It is argued that colors used in the textiles actually affect emissivity more than the charging elements. Notice that the test used to achieve this result required some nonstandard procedure due to the semitransparent nature of textiles as opposed to conventional techniques for opaque surfaces.
In the area of buildings and infrastructures infrared applications, S. Caglio et al. focus on an infrared thermography procedure for heat diffusion in ceramics finishing materials of contemporary architecture. The aim of the research is the development of a method for the thermal characterization of clinkers, a very common ceramic finishing material of buildings. To this end, they analyze correlation between parameters found by thermography and the damage level in the specimens; a Gio Ponti building is discussed as case study.

Finally, in the area of remote sensing, P. Coppo et al. describe a simplified end-to-end software tool for simulation of data produced by optical instruments, starting from either synthetic or airborne hyperspectral data. Some simulation examples of hyper spectral and panchromatic images for existing and future design instruments are also reported. Both high spatial/spectral resolution images with low intrinsic noise and the sensor/mission specifications are used as inputs for simulations, thus showing the tool capabilities in simulating target detection scenario, including data quality assessment and class discrimination, as well as in understanding the impact of optical design on image quality.

\section{Acknowledgments}

AITA Conference in L'Aquila as well as all the previous AITA Conferences were enjoying the support and participation of a great scientist and overall a great man, Ermanno Grinzato. Ermanno, one of the best examples of the Veneto's culture, was teaching us as real man should be: honest, sincere, and lovers of life and any good aspects of it. Probably life, due to the exchange of this love, was calling him too early, leaving us and to all the world his great lesson of sincerity, honesty, and love for the good things. For this all of us are missing him and his love for the important aspects of life. Arrivederci Ermanno and thanks for your great lesson.

Ovidio Salvetti
Laura Abbozzo Ronchi
Carlo Corsi
Antoni Rogalski
Marija Strojnik



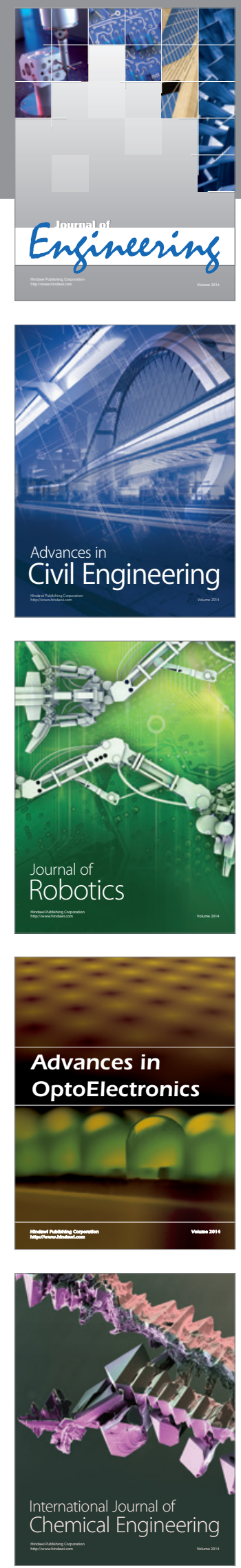

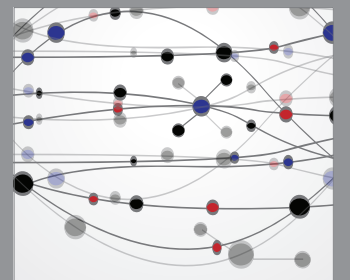

The Scientific World Journal
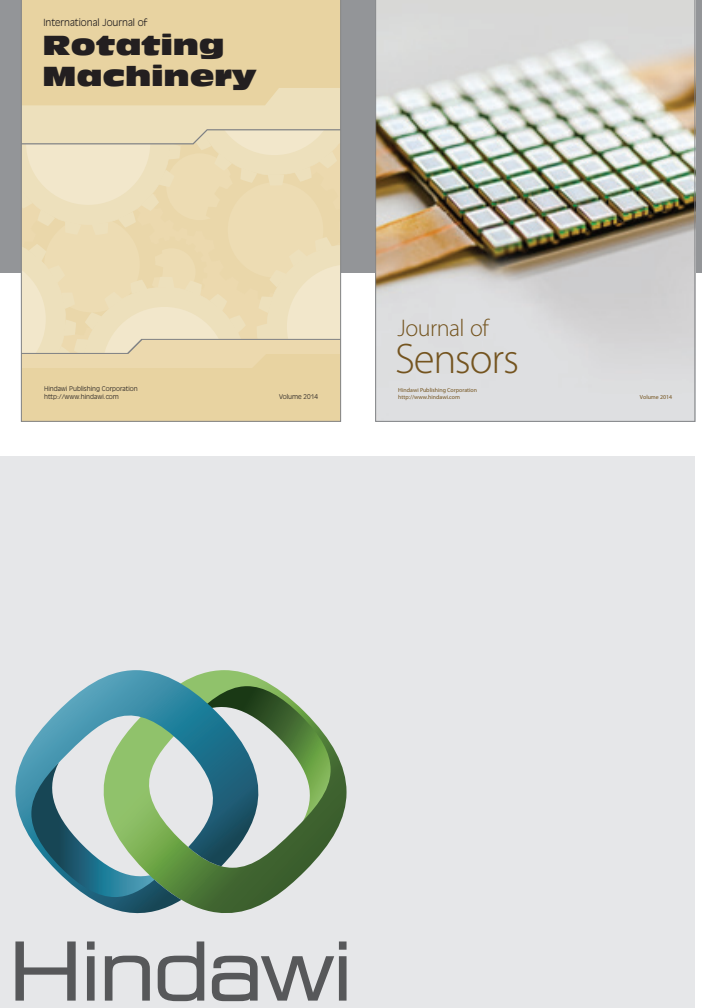

Submit your manuscripts at http://www.hindawi.com
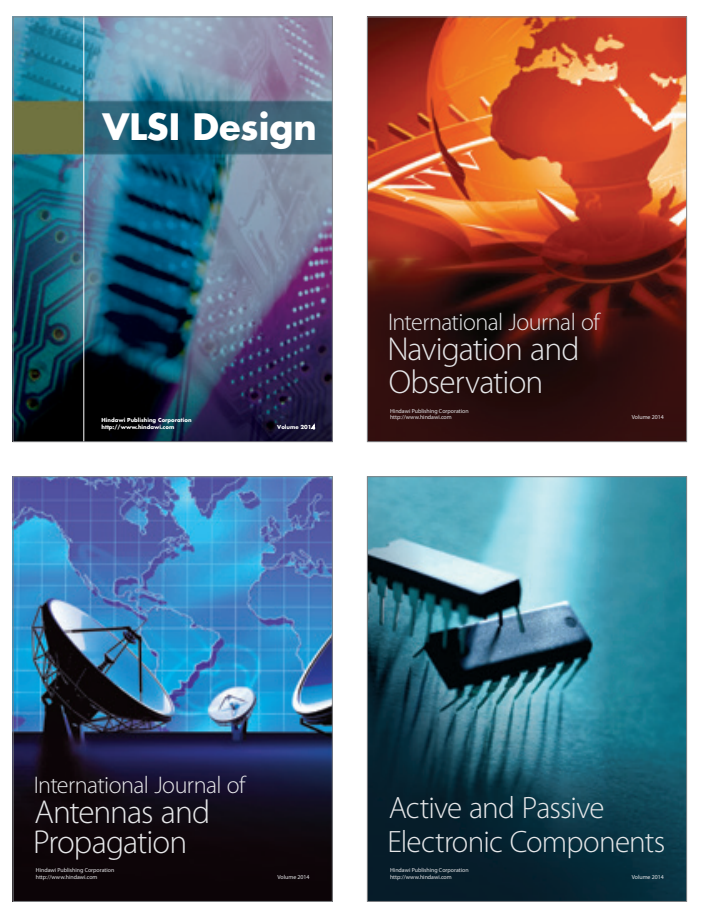
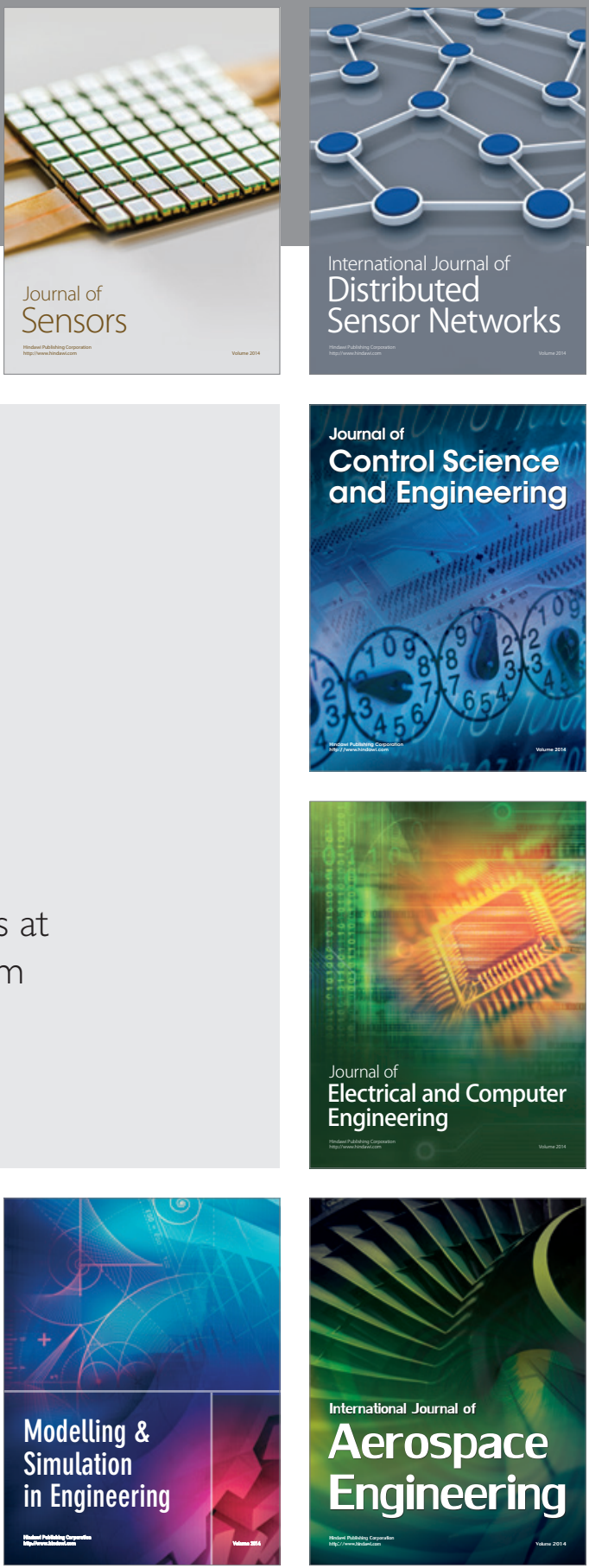

Journal of

Control Science

and Engineering
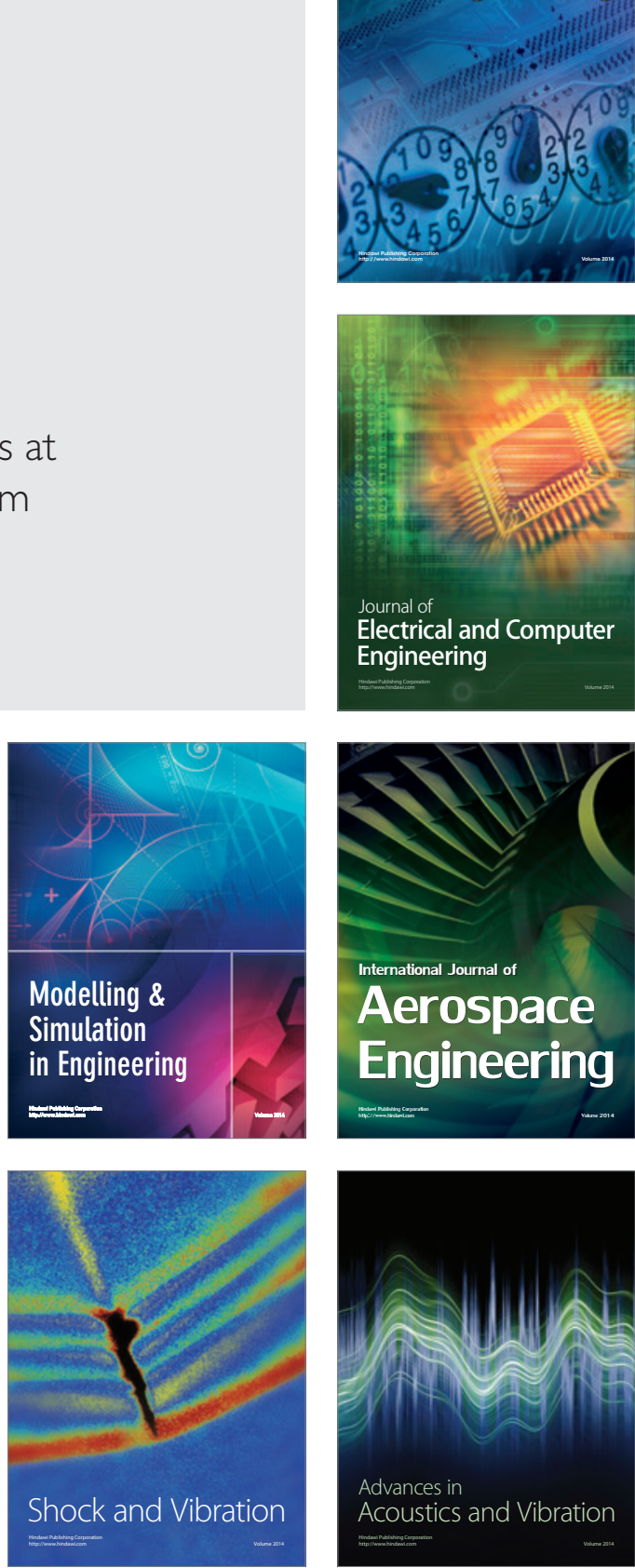